\title{
TANGENT BUNDLE ENDOWED WITH QUARTER-SYMMETRIC NON-METRIC CONNECTION ON AN ALMOST HERMITIAN MANIFOLD
}

\author{
Mohammad Nazrul Islam Khan
}

(C) 2020 by University of Niš, Serbia | Creative Commons License: CC BY-NC-ND

\begin{abstract}
In this paper, we have studied the tangent bundle endowed with quartersymmetric non-metric connection obtained by vertical and complete lifts of a quartersymmetric non-metric connection on the base manifold and, also, proposed the study of the tangent bundle of an almost Hermitian manifold and an almost Kaehler manifold. Finally, we obtained some theorems for Nijenhuis tensor on the tangent bundle of an almost Hermitian manifold and an almost Kaehler manifold.

Keywords: Almost Hermitian manifold, almost Kaehler manifold, vertical lift, complete lift, Nijenhuis tensor.
\end{abstract}

\section{Introduction}

The idea of quarter-symmetric linear connections in differentiable manifold was introduced by Golab [4] in 1975. A linear connection is said to be a quartersymmetric connection if its torsion tensor $\widetilde{T}$ is of the for

$$
\widetilde{T}(X, Y)=u(Y) \phi X-u(X) \phi Y
$$

where $u$ is 1 -form and $\phi$ is a tensor of type $(1,1)$. Agashe and Chafle [1] studied a semi-symmetric non-metric connection on a Riemannian manifold in 1992. In 2007, the author [7] defined and studied a quarter-symmetric semi-metric connection on Sasakian manifold. In 2008, Chaturvedi and Pandey [2] studied Kaehler manifold equipped with a semi-symmetric non-metric connection.

The method of lift has an important role in modern differential geometry. With the lift function, it is possible to generalize to differentiable structures on any manifold to its extensions. The complete, vertical and horizontal lifts of tensor fields and connections on any manifold $M$ to the tangent manifold $T M$ were obtained by Yano and Ishihara [13] in 1973. In 1969, Tani [11] developed the theory of

Received February 3, 2019; accepted September 16, 2019

2010 Mathematics Subject Classification. Primary 53C15, 53C35; Secondary 53B15, 53B35 
hypersurfaces prolonged to tangent bundle with respect to complete lift of metric tensor of Riemannian manifold. In 2005, Das and the author [3] obtained almost product structure by means of the complete, vertical and horizontal lifts of almost r-contact structures on the tangent bundle. The author [8] studied the lifts of hypersurfaces with quarter-symmetric semi-metric connection to the tangent bundles and obtained an important result (Theorem3 in [8]). We have used similar method in section 3 of this paper. Among some other authors who studied the differential geometry of the tangent bundle are the following $[6,9,12,14]$.

The paper is structured as follows. In Section 2, we will recall an almost Hermitian manifold, Quarter symmetric non-metric connection, Tangent bundle, Induced metric and connection. We will consider, in Section 3, the tangent bundle endowed with quarter-symmetric non-metric connection obtained by vertical and complete lifts of a quarter-symmetric non-metric connection on the base manifold and propose to study the tangent bundle of an almost Hermitian manifold and an almost Kaehler manifold. In Section 4, we will obtain some theorems for Nijenhuis tensor on tangent bundle of an almost Hermitian manifold and an almost Kaehler manifold. In the last Section, we will construct an example of a four dimensional almost Hermitian manifold on tangent bundle.

\section{Preliminaries}

\subsection{An almost Hermitian manifold}

A tensor field $\hat{F}$ of type $(1,1)$ on an even dimensional differentiable manifold $M, n=2 m$ such that

$$
\text { (2.1) } \hat{F}^{2} \hat{X}+\hat{X}=0 \text {. }
$$

If non-singular Hermitian metric of type $(0,2)$ satisfies

$$
\hat{g}(\hat{F} \hat{X}, \hat{F} \hat{Y})=\hat{g}(\hat{X}, \hat{Y})
$$

for arbitrary vector fields $\hat{X}, \hat{Y}$, then $(M, \hat{F}, \hat{g})$ is called an almost Hermitian manifold with an almost Hermitian structure $(\hat{F}, \hat{g})$. Let $\hat{\nabla}$ be the Riemannian connection on $M$, then $M$ is said to be a Kaehler manifold [2] if

$$
\left(\hat{\nabla}_{\hat{X}} \hat{F}\right) \hat{Y}=0 .
$$

\subsection{Quarter-symmetric non-metric connection}

A linear connection $\bar{\nabla}$ on $M$ is defined as

$$
\bar{\nabla}_{\hat{X}} \hat{Y}=\hat{\nabla}_{\hat{X}} \hat{Y}+\hat{\omega}(\hat{Y}) \hat{F} \hat{X},
$$

for arbitrary vector fields $\hat{X}, \hat{Y}, 1$-form $\hat{\omega}$ and $\hat{\nabla}$ denotes Riemannian connection on $M$.

The torsion tensor $\bar{T}$ is given by 


$$
\bar{T}(\hat{X}, \hat{Y})=\bar{\nabla}_{\hat{X}} \hat{Y}+\bar{\nabla}_{\hat{Y}} \hat{X}-[\hat{X}, \hat{Y}] .
$$

The connection $\bar{\nabla}$ is symmetric if its torsion tensor vanishes, otherwise, it is nonsymmetric. If there is a metric $\hat{g}$ in $M$ such that $\bar{\nabla} \hat{g}=0$, then the connection $\bar{\nabla}$ is a metric connection; otherwise it is non-metric [5].

A linear connection $\bar{\nabla}$ is said to be a quarter-symmetric linear connection if its torsion connection $\bar{T}$ is of the form

$$
\bar{T}(\hat{X}, \hat{Y})=\hat{\omega}(\hat{Y}) \hat{F} \hat{X}-\hat{\omega}(\hat{X}) \hat{F} \hat{Y}
$$

and

$$
\left(\bar{\nabla}_{\hat{X}} \hat{g}\right)(\hat{X}, \hat{Y})=-\hat{\omega}(\hat{Y}) \hat{g}(\hat{F} \hat{X}, \hat{Z})-\hat{\omega}(\hat{Z}) \hat{g}(\hat{F} \hat{X}, \hat{Y}) .
$$

The connection $\bar{\nabla}$ satisfying (2.4), (2.5) and(2.6) is called quarter-symmetric non-metric connection [7].

\subsection{Tangent Bundle}

Let $T_{p}(M)$ be the tangent space of differentiable manifold $M$ at a point of $M$, then the set $T(M)=\cup_{p \in M} T_{p}(M)$ is called the tangent bundle over the manifold $M$. For any point $\widetilde{p}$ of $T(M)$, the correspondence $\widetilde{p} \rightarrow p$ determines the bundle projection $\pi: T(M) \rightarrow M$. Thus $\pi(\widetilde{p})=p$, where $\pi: T(M) \rightarrow M$ defines the bundle projection of $T(M)$ over $M$. The set $\pi^{-1}(p)$ is called the fibre over $p \in M$ and $M$ the base space $[3,13]$.

Vertical lifts: If $f$ is a function in $M$, we write $f^{V}$ for the function in $T(M)$ obtained by forming the composition of $\pi: T(M) \rightarrow M$ and $f: M \rightarrow M$, so that $f^{V}=f o \pi$. Thus, if a point $\widetilde{p} \in \pi^{-1}(U)$ has induced coordinates $\left(x^{h}, y^{h}\right)$, then $f^{V}(\widetilde{p})=f^{V}(x, y)=f o \pi(\widetilde{p})=f(p)=f(x)$ thus the value of $f^{V}(\widetilde{p})$ is constant along each fibre $T_{p}(M)$ and equal to the value $f(p)$. We call $f^{V}$ the vertical lift of the function $f$.

Complete lifts: If $f$ is a function in $M$, we write $f^{C}$ for the function in $T(M)$ defined by $f^{C}=i(d f)$ and call $f^{C}$ the complete lift of the function $f$. The complete lift $f^{C}$ of a function $f$ has the local expression $f^{C}=y^{i} \partial_{i} f=\partial f$ with respect to the induced coordinates in $T(M)$, where $\partial f$ denotes $y^{i} \partial_{i} f$.

Suppose that $\hat{X} \in \operatorname{Im}_{0}^{1}(M)$. We define a vector field $\hat{X}^{C}$ in $T(M)$ by $\hat{X}^{C} f^{C}=$ $(\hat{X} f)^{C}, f$ being an arbitrary function in $M$ and call $\hat{X}^{C}$ the complete lift of call $\hat{X}$ in $T(M)$.

Suppose that $\hat{\omega} \in \operatorname{Im}_{0}^{1}(M)$. Then a 1 -form $\hat{\omega}^{C}$ in $T(M)$ defined by $\hat{\omega}^{C}\left(\hat{X}^{C}\right)=$ $(\hat{\omega}(\hat{X}))^{C}, \hat{X}$ being an arbitrary vector field in $M$. We call $\hat{\omega}^{C}$ the complete lift of $\hat{\omega}$. Moreover, these lifts have the following properties [8]: 


$$
\begin{gathered}
{\left[\hat{X}^{C}, \hat{Y}^{C}\right]=[\hat{X}, \hat{Y}]^{C} ; \hat{F}^{C}\left(\hat{X}^{C}\right)=(\hat{F}(\hat{X}))^{C}} \\
\hat{\omega}^{V}\left(\hat{X}^{C}\right)=(\hat{\omega}(\hat{X}))^{V} ; \hat{\omega}^{C}\left(\hat{X}^{C}\right)=(\hat{\omega}(\hat{X}))^{C} \\
\hat{g}^{C}\left(\hat{X}^{V}, \hat{Y}^{C}\right)=\hat{g}^{C}\left(\hat{X}^{C}, \hat{Y}^{V}\right)=(\hat{g}(\hat{X}, \hat{Y}))^{V} ; \hat{g}^{C}\left(\hat{X}^{V}, Y^{C}\right)=(\hat{g}(\hat{X}, \hat{Y}))^{C} \\
\hat{T}^{C}\left(\hat{X}^{C}, \hat{Y}^{C}\right)=(\hat{T}(\hat{X}, \hat{Y}))^{C}
\end{gathered}
$$

where $\hat{X}^{V}, \hat{\omega}^{V}, \hat{g}^{V}, \hat{T}^{V}$ and $\hat{X}^{C}, \hat{\omega}^{C}, \hat{g}^{C}, \hat{T}^{C}$ are vertical and complete lifts of $\hat{X}, \hat{\omega}, \hat{g}, \hat{T}$ respectively.

\subsection{Induced metric and connection on $T(S)$}

Let $S$ be a manifold of $(n-1)$-dimension immersed in $M$ by the immersion $\tau: S \rightarrow M$. Let us denote the differentiable mapping $d \tau$ of the immersion $\tau$ of $B$ is a mapping from $T S$ into $T M$, which is called tangent map of $\tau$ where $T(S)$ and $T(M)$ are the tangent bundles of $S$ into $M$ respectively. The tangent map of $B$ is denoted by $\widetilde{B}: T(T(S)) \rightarrow T(T(M))$.

Let $\hat{g}$ be the Riemannian metric in $M$. The complete lift $\hat{g}^{C}$ of $\hat{g}$ in $T(M)$. If we denote by $\widetilde{g}$ the induced metric on $T(S)$ from $\hat{g}^{C}$ then we have $\widetilde{g}\left(X^{C}, Y^{C}\right)=$ $\hat{g}^{C}\left(\tilde{B} X^{C}, \widetilde{B} Y^{C}\right)$ for $X, Y \in \operatorname{Im}_{0}^{1}(S)$. The complete lift $\hat{\nabla}^{C}$ of $\hat{\nabla}$ to $T(M)$ have the property $\hat{\nabla}_{\hat{X}^{C}}^{C} \hat{Y}^{C}=\left(\hat{\nabla}_{\hat{X}} \hat{Y}\right)^{C} ; \hat{\nabla}_{\hat{X}^{C}}^{C} \hat{Y}^{V}=\left(\hat{\nabla}_{\hat{X}} \hat{Y}\right)^{V}$ for $X, Y \in \operatorname{Im}_{0}^{1}(M), \hat{\nabla}$ being Riemannian connection of $T(M)$ with respect to $\hat{g}, \hat{\nabla}^{C}$ is Riemannian connection of $T(M)$ with respect to $\hat{g}^{C}$ [14]. Similarly, the complete lift $\hat{\nabla}^{C}$ of the induce connection $\nabla$ on $(S, g)$ is also the Riemannian connection in $(T(M), \widetilde{g})$.

Yano and Ishihara [13] proved the following theorem:

THEOREM 2.1. If $\hat{T}$ is torsion tensor of $\hat{\nabla}^{C}$ in $(M, \hat{g})$, then $\hat{T}^{C}$ is torsion tensor of $\hat{\nabla}^{C}$ in $(T(M), \hat{g})$.

In [11], using (3.10) we have

$$
\begin{aligned}
& \hat{\omega}^{V}\left(\widetilde{B} X^{C}\right)=\hat{\omega}^{V}(B X)^{\bar{C}}=\hbar\left(\hat{\omega}^{V}\left(\hat{X}^{C}\right)\right)=\hbar(\hat{\omega}(\hat{X}))^{V}=(\hat{\omega}(B X))^{\bar{V}} \\
& \hat{\omega}^{C}\left(\widetilde{B} X^{C}\right)=\hat{\omega}^{C}(B X)^{\bar{C}}=\hbar\left(\hat{\omega}^{C}\left(\hat{X}^{C}\right)\right)=\hbar(\hat{\omega}(\hat{X}))^{C}=(\hat{\omega}(B X))^{C}
\end{aligned}
$$

for arbitrary vector fields $X, Y$ in $S$. Here, we denote the operation of restriction to $\pi_{M}^{-1}(\tau(S))$ by $\hbar$ and the vertical and complete lift operations on $\pi_{M}^{-1}(\tau(S))$ by $\bar{V}$ and $\bar{C}$ respectively.

\section{Tangent bundle endowed with quarter-symmetric non-metric connection on an almost Hermitian manifold}

Let $M$ be an almost Hermitian manifold and $T(M)$ be its tangent bundle. The complete lift of the $\hat{F}$ and $\hat{g}$ are $\hat{F}^{C}$ and $\hat{g}^{C}$ satisfying

$$
\left(\hat{F}^{C}\right)^{2} \hat{X}+\hat{X}=0 .
$$




$$
\hat{g}^{C}\left((\hat{F} \hat{X})^{C},(\hat{F} \hat{Y})^{C}\right)=\hat{g}^{C}\left(\widetilde{B} X^{C}, \widetilde{B} Y^{C}\right)
$$

An almost Hermitian manifold $M$ is called a Kaehler manifold if

$$
\left(\hat{\nabla}_{\widetilde{B} X^{C}}^{C} \widetilde{B} F^{C}\right)\left(\widetilde{B} Y^{C}, \widetilde{B} Z^{C}\right)=0
$$

a Nearly Kaehler manifold if

$$
\left(\hat{\nabla}_{\widetilde{B} X^{C}}^{C} \widetilde{B} F^{C}\right)\left(\widetilde{B} Y^{C}, \widetilde{B} Z^{C}\right)=\left(\hat{\nabla}_{\widetilde{B} Y^{C}}^{C} \widetilde{B} F^{C}\right)\left(\widetilde{B} Z^{C}, \widetilde{B} X^{C}\right),
$$

an almost Kaehler manifold if

$$
\begin{array}{r}
\left(\hat{\nabla}_{\widetilde{B} X^{C}}^{C} \widetilde{B} F^{C}\right)\left(\widetilde{B} Y^{C}, \widetilde{B} Z^{C}\right)+\left(\hat{\nabla}_{\widetilde{B} Y^{C}}^{C} \widetilde{B} F^{C}\right)\left(\widetilde{B} Z^{C}, \widetilde{B} X^{C}\right) \\
+\left(\hat{\nabla}_{\widetilde{B} Z^{C}}^{C} \widetilde{B} F^{C}\right)\left(\widetilde{B} X^{C}, \widetilde{B} Y^{C}\right)=0,
\end{array}
$$

a Quasi-Kaehler manifold if

$$
\left(\hat{\nabla}_{\widetilde{B}(F X)^{C}}^{C} \widetilde{B} F^{C}\right)\left(\widetilde{B}(F Y)^{C}, \widetilde{B} Z^{C}\right)+\left(\hat{\nabla}_{\widetilde{B} X^{C}}^{C} \widetilde{B} F^{C}\right)\left(\widetilde{B} Y^{C}, \widetilde{B} Z^{C}\right)=0
$$

for arbitrary vector fields $X, Y, Z$ and where $\hat{\nabla}^{C}$ is the Riemannian connection of $T(M)$.

If we define

$$
{ }^{\prime} F^{C}\left(\widetilde{B} X^{C}, \widetilde{B} Y^{C}\right)=\hat{g}^{C}\left(\widetilde{B}(F X)^{C}, \widetilde{B}(F Y)^{C}\right)
$$

taking the complete lift on the both sides of the equation (2.4) we get,

$$
\begin{gathered}
\left(\bar{\nabla}_{B X} B Y\right)^{\bar{C}}=(\hat{\nabla} \widetilde{B} X \widetilde{B} Y)^{\bar{C}}+(\hat{\omega}(B X)(B F Y))^{\bar{C}} \\
(3.8) \bar{\nabla}_{\widetilde{B} X^{C}} \widetilde{B} Y^{C}=\hat{\nabla}_{\widetilde{B} X^{C}} \widetilde{B} Y^{C}+\hat{\omega}^{C}\left(\widetilde{B} Y^{C}\right)\left(\widetilde{B}(F X)^{V}\right)+\hat{\omega}^{V}\left(\widetilde{B} Y^{V}\right)\left(\widetilde{B}(F X)^{C}\right)
\end{gathered}
$$

Now,

$$
\begin{aligned}
\bar{\nabla}_{\widetilde{B} X^{C}}^{C} \widetilde{B} Y^{C}-\bar{\nabla}_{\widetilde{B} Y^{C}}^{C} \widetilde{B} X^{C}-\left[X^{C}, Y^{C}\right] & =\hat{\nabla}_{\widetilde{B} X^{C}}^{C} \widetilde{B} Y^{C} \\
& +\hat{\omega}^{C}\left(\widetilde{B} Y^{C}\right)\left(\widetilde{B}(F X)^{V}\right)+\hat{\omega}^{V}\left(\widetilde{B} Y^{V}\right)\left(\widetilde{B}(F X)^{C}\right) \\
& -\hat{\nabla}_{\widetilde{B} Y^{C}}^{C} \widetilde{B} X^{C}-\hat{\omega}^{C}\left(\widetilde{B} X^{C}\right)\left(\widetilde{B}(F Y)^{V}\right) \\
& -\hat{\omega}^{V}\left(\widetilde{B} X^{V}\right)\left(\widetilde{B}(F Y)^{C}\right)-\left[X^{C}, Y^{C}\right] .
\end{aligned}
$$

Using the theorem 2.1, we get

$$
\text { (3.9) } \begin{aligned}
\bar{T}^{C}\left(\widetilde{B} X^{C}, \widetilde{B} Y^{C}\right) & =\hat{\omega}^{C}\left(\widetilde{B} Y^{C}\right)\left(\widetilde{B}(F X)^{V}\right)+\hat{\omega}^{V}\left(\widetilde{B} Y^{V}\right)\left(\widetilde{B}(F X)^{C}\right) \\
& -\hat{\omega}^{C}\left(\widetilde{B} X^{C}\right)\left(\widetilde{B}(F Y)^{V}\right)-\hat{\omega}^{V}\left(\widetilde{B} X^{V}\right)\left(\widetilde{B}(F Y)^{C}\right),
\end{aligned}
$$


and

$$
\begin{aligned}
\left(\bar{\nabla}_{\widetilde{B} X^{C}}^{C}\right)\left(\widetilde{B} Y^{C}, \widetilde{B} Z^{C}\right) & =(\hat{\omega}(\widetilde{B} Y))^{C}(\hat{g}(\widetilde{B}(F X), \widetilde{B} Z))^{V} \\
+ & (\hat{\omega}(\widetilde{B} Y))^{V}(\hat{g}(\widetilde{B}(F X), \widetilde{B} Z))^{C} \\
+ & (\hat{\omega}(\widetilde{B} Z))^{C}(\hat{g}(\widetilde{B}(Y), \widetilde{B}(F X)))^{V} \\
+ & (\hat{\omega}(\widetilde{B} Z))^{V}(\hat{g}(\widetilde{B}(Y), \widetilde{B}(F X)))^{C}
\end{aligned}
$$

The equation (3.8) can be written as

$$
\bar{\nabla}_{\widetilde{B} X^{C}} \widetilde{B} Y^{C}=\hat{\nabla}_{\widetilde{B} X^{C}} \widetilde{B} Y^{C}+H(X, Y),
$$

where

$$
H(X, Y)=\hat{\omega}^{C}\left(\widetilde{B} Y^{C}\right)\left(\widetilde{B}(F X)^{V}\right)+\hat{\omega}^{V}\left(\widetilde{B} Y^{V}\right)\left(\widetilde{B}(F X)^{C}\right) .
$$

If we define

$$
{ }^{\prime} H(X, Y, Z)=\hat{g}^{C}\left(H(X, Y), \widetilde{B} Z^{C}\right)
$$

then in the view of $(3.12),(3.13)$ becomes

$$
\begin{aligned}
{ }^{\prime} H(X, Y, Z) & =(\hat{\omega}(\widetilde{B} Y))^{C}(\hat{g}(\widetilde{B}(F X), \widetilde{B} Z))^{V} \\
& +(\hat{\omega}(\widetilde{B} Y))^{V}(\hat{g}(\widetilde{B}(F X), \widetilde{B} Z))^{C} .
\end{aligned}
$$

Theorem 3.1. If an almost Hermitian manifold $M$ admits a quarter symmetric non-metric connection $\bar{\nabla}^{C}$ with respect to the Riemannian connection $\hat{\nabla}^{C}$ in $\left(T(M), \hat{g}^{C}\right)$, then the necessary and sufficient condition for an almost Hermitian manifold to be a Hermitian manifold is that metric connection $\left(\hat{\nabla}_{\widetilde{B} X^{C}}^{C} \widetilde{B} F^{C}\right)\left(\widetilde{B} Y^{C}\right)$ is hybrid in both slots, i.e. $\left(\hat{\nabla}_{\widetilde{B}(F X)^{C}}^{C} \widetilde{B} F^{C}\right)\left(\widetilde{B}(F Y)^{C}=\left(\hat{\nabla}_{\widetilde{B} X^{C}}^{C} \widetilde{B} F^{C}\right)\left(\widetilde{B} Y^{C}\right)\right.$.

Proof. Covariant derivative of $(F Y)^{C}$ with respect to the connection $\bar{\nabla}^{C}$ gives

$$
\left(\bar{\nabla}_{\tilde{B} X^{C}}^{C} \widetilde{B} F^{C}\right)\left(\widetilde{B} Y^{C}\right)+F^{C}\left(\bar{\nabla}_{\tilde{B} X^{C}}^{C} \widetilde{B} Y^{C}\right)=\bar{\nabla}_{\widetilde{B} X^{C}}^{C} \widetilde{B}(F Y)^{C}
$$

In consequence of (3.1) and (3.8), the last expression becomes

$$
\begin{aligned}
\left(\bar{\nabla}_{\widetilde{B} X^{C}}^{C} \widetilde{B} F^{C}\right)\left(\widetilde{B} Y^{C}\right) & =\left(\hat{\nabla}_{\widetilde{B} X^{C}}^{C} \widetilde{B} F^{C}\right)\left(\widetilde{B} Y^{C}\right)+\hat{\omega}^{C}\left(\widetilde{B} Y^{C}\right) \widetilde{B} X^{V} \\
& +\hat{\omega}^{V}\left(\widetilde{B} Y^{V}\right) \widetilde{B} X^{C}+\hat{\omega}^{C}\left(\widetilde{B}(F Y)^{C}\right)\left(\widetilde{B}(F X)^{V}\right) \\
& +\hat{\omega}^{V}\left(\widetilde{B}(F Y)^{V}\right)\left(\widetilde{B}(F X)^{C}\right) .
\end{aligned}
$$

Replacing $\mathrm{X}$ by FX and $\mathrm{Y}$ by FY in (3.15) and using (3.1), we get

$$
\left(\bar{\nabla}_{\widetilde{B}(F X)^{C}}^{C} \widetilde{B} F^{C}\right)\left(\widetilde{B}(F Y)^{C}\right)=\left(\hat{\nabla}_{\widetilde{B}(F X)^{C}}^{C} \widetilde{B} F^{C}\right)\left(\widetilde{B}(F Y)^{C}\right)
$$




$$
\begin{aligned}
& +\hat{\omega}^{C}\left(\widetilde{B}(F Y)^{C}\right) \widetilde{B}(F X)^{V} \\
& +\hat{\omega}^{C}\left(\widetilde{B} Y^{C}\right) \widetilde{B} X^{V} \\
& +\hat{\omega}^{V}\left(\widetilde{B}(F Y)^{V}\right) \widetilde{B}(F X)^{C} \\
& +\hat{\omega}^{V}\left(\widetilde{B} Y^{V}\right) \widetilde{B} X^{C} .
\end{aligned}
$$

Subtracting (3.15) from (3.16), we have

$$
\begin{aligned}
\left(\bar{\nabla}_{\widetilde{B}(F X)^{C}}^{C} \widetilde{B} F^{C}\right)\left(\widetilde{B}(F Y)^{C}\right) & -\left(\bar{\nabla}_{\widetilde{B} X^{C}}^{C} \widetilde{B} F^{C}\right)\left(\widetilde{B} Y^{C}\right) \\
& =\left(\hat{\nabla}_{\widetilde{B}(F X)^{C}}^{C} \widetilde{B} F^{C}\right)\left(\widetilde{B}(F Y)^{C}\right) \\
& -\left(\hat{\nabla}_{\widetilde{B} X^{C}}^{C} \widetilde{B} F^{C}\right)\left(\widetilde{B} Y^{C}\right) .
\end{aligned}
$$

A necessary and sufficient condition for an almost Hermitian manifold to be a Hermitian manifold is

$$
\left(\hat{\nabla}_{\widetilde{B}(F X)^{C}}^{C} \widetilde{B} F^{C}\right)\left(\widetilde{B}(F Y)^{C}\right)=\left(\hat{\nabla}_{\widetilde{B} X^{C}}^{C} \widetilde{B} F^{C}\right)\left(\widetilde{B} Y^{C}\right)
$$

In view of (3.17) and (3.18), we obtain the statement of the theorem.

Theorem 3.2. An almost Hermitian manifold $M$ admits a quarter-symmetric non-metric connection $\bar{\nabla}^{C}$ with respect to the Riemannian connection $\hat{\nabla}^{C}$ in $\left(T(M), \hat{g}^{C}\right)$ is an almost Kaehler manifold if and only if ${ }^{\prime} F^{C}$ is closed with respect to the connection $\bar{\nabla}^{C}$.

Proof. We have

$$
\begin{aligned}
X^{C}\left({ }^{\prime} F^{C}\left(\widetilde{B} Y^{C}, \widetilde{B} Z^{C}\right)\right)= & \bar{\nabla}_{\widetilde{B} X^{C}}^{C} \widetilde{B}^{\prime} F^{C}\left(\widetilde{B} Y^{C}, \widetilde{B} Z^{C}\right) \\
& +{ }^{\prime} F^{C}\left(\bar{\nabla}_{\widetilde{B} X^{C}}^{C} \widetilde{B} Y^{C}, \widetilde{B} Z^{C}\right) \\
& +{ }^{\prime} F^{C}\left(\widetilde{B} Y^{C}, \bar{\nabla} \widetilde{B} X^{C} \widetilde{B} Z^{C}\right) \\
= & \hat{\nabla}_{\widetilde{B} X^{C}}^{C} \widetilde{B}^{\prime} F^{C}\left(\widetilde{B} Y^{C}, \widetilde{B} Z^{C}\right) \\
& +{ }^{\prime} F^{C}\left(\hat{\nabla}_{\widetilde{B} X^{C}}^{C} \widetilde{B} Y^{C}, \widetilde{B} Z^{C}\right) \\
& +{ }^{\prime} F^{C}\left(\widetilde{B} Y^{C}, \hat{\nabla}_{\widetilde{B} X^{C}}^{C} \widetilde{B} Z^{C}\right),
\end{aligned}
$$

then

$$
\begin{array}{rc}
\left(\bar{\nabla}_{\widetilde{B} X^{C}}^{C} \widetilde{B}^{\prime} F^{C}\right)\left(\widetilde{B} Y^{C}, \widetilde{B} Z^{C}\right) & =\hat{\nabla}_{\widetilde{B} X^{C}}^{C} \widetilde{B}^{\prime} F^{C}\left(\widetilde{B} Y^{C}, \widetilde{B} Z^{C}\right) \\
& { }^{\prime} F^{C}\left(\bar{\nabla}_{\widetilde{B} X^{C}}^{C} \widetilde{B} Y^{C}-\hat{\nabla}_{\widetilde{B} X^{C}}^{C} \widetilde{B} Y^{C}, \widetilde{B} Z^{C}\right) \\
& -^{\prime} F^{C}\left(\widetilde{B} Y^{C}, \bar{\nabla}_{\widetilde{B} X^{C}}^{C} \widetilde{B} Y^{C}-\hat{\nabla}_{\widetilde{B} X^{C}}^{C} \widetilde{B} Z^{C}\right) .
\end{array}
$$

In consequence of $(3.1),(3.2),(3.8)$, the last expression becomes

$$
\left(\bar{\nabla}_{\widetilde{B} X^{C}}^{C} \widetilde{B}^{\prime} F^{C}\right)\left(\widetilde{B} Y^{C}, \widetilde{B} Z^{C}\right) \quad=\hat{\nabla}_{\widetilde{B} X^{C}}^{C} \widetilde{B}^{\prime} F^{C}\left(\widetilde{B} Y^{C}, \widetilde{B} Z^{C}\right)
$$




$$
\begin{gathered}
+\hat{\omega}^{C}\left(\widetilde{B} Y^{C}\right) \hat{g}^{C}\left(\widetilde{B}(X)^{V}, \widetilde{B}(Z)^{C}\right) \\
+\hat{\omega}^{V}\left(\widetilde{B} Y^{V}\right) \hat{g}^{C}\left(\widetilde{B}(X)^{C}, \widetilde{B}(Z)^{C}\right) \\
-\hat{\omega}^{C}\left(\widetilde{B} Z^{C}\right) \hat{g}^{C}\left(\widetilde{B}(F Y)^{C}, \widetilde{B}(F X)^{V}\right) \\
-\hat{\omega}^{V}\left(\widetilde{B} Z^{V}\right) \hat{g}^{C}\left(\widetilde{B}(F Y)^{C}, \widetilde{B}(F X)^{C}\right)
\end{gathered}
$$

Taking the cycle sum of (3.19) in X, Y, Z, we get

$$
\begin{aligned}
\left(\bar{\nabla}_{\widetilde{B} X^{C}}^{C} \widetilde{B}^{\prime} F^{C}\right)\left(\widetilde{B} Y^{C}, \widetilde{B} Z^{C}\right) & +\left(\bar{\nabla}_{\widetilde{B} Y^{C}}^{C} \widetilde{B}^{\prime} F^{C}\right)\left(\widetilde{B} Z^{C}, \widetilde{B} X^{C}\right) \\
& +\left(\bar{\nabla}_{\widetilde{B} Z^{C}}^{C} \widetilde{B}^{\prime} F^{C}\right)\left(\widetilde{B} X^{C}, \widetilde{B} Y^{C}\right) \\
& =\hat{\nabla}_{\widetilde{B} X^{C}}^{C} \widetilde{B}^{\prime} F^{C}\left(\widetilde{B} Y^{C}, \widetilde{B} Z^{C}\right) \\
& +\hat{\nabla}_{\widetilde{B} Y^{C}}^{C} \widetilde{B}^{\prime} F^{C}\left(\widetilde{B} Z^{C}, \widetilde{B} X^{C}\right) \\
& +\hat{\nabla}_{\widetilde{B} Z^{C}}^{C} \widetilde{B}^{\prime} F^{C}\left(\widetilde{B} X^{C}, \widetilde{B} Y^{C}\right) .
\end{aligned}
$$

In consequence of (3.5) and (3.20), we see that ${ }^{\prime} F^{C}$ is closed with respect to the connection $\bar{\nabla}^{C}$. Converse part is obvious from (3.20).

\section{Theorems on Nijenhuis Tensor with $M$ admits a quarter symmetric non-metric connection $\bar{\nabla}^{C}$}

Theorem 4.1. An almost Hermitian manifold $M$ admits a quarter-symmetric non-metric connection $\bar{\nabla}^{C}$ with respect to the Riemannian connection $\hat{\nabla}^{C}$ in $\left(T(M), \hat{g}^{C}\right)$ then the Nijenhuis Tensor of $\hat{\nabla}^{C}$ and $\bar{\nabla}^{C}$ coincide.

Proof. From (3.15), we have

$$
\begin{aligned}
\left(\hat{\nabla}_{\widetilde{B} X^{C}}^{C} \widetilde{B} F^{C}\right)\left(\widetilde{B} Y^{C}\right) & =\left(\bar{\nabla}_{\widetilde{B} X^{C}}^{C} \widetilde{B} F^{C}\right)\left(\widetilde{B} Y^{C}\right)-\hat{\omega}^{C}\left(\widetilde{B} Y^{C}\right) \widetilde{B} X^{V} \\
& -\hat{\omega}^{V}\left(\widetilde{B} Y^{V}\right) \widetilde{B} X^{C}-\hat{\omega}^{C}\left(\widetilde{B}(F Y)^{C}\right)\left(\widetilde{B}(F X)^{V}\right) \\
& -\hat{\omega}^{V}\left(\widetilde{B}(F Y)^{V}\right)\left(\widetilde{B}(F X)^{C}\right) .
\end{aligned}
$$

Replacing $X$ by $F X$ in (4.1) and using (3.1), we find

$$
\begin{aligned}
\left(\hat{\nabla}_{\widetilde{B}(F X)^{C}}^{C} \widetilde{B} F^{C}\right)\left(\widetilde{B} Y^{C}\right) & =\left(\bar{\nabla}_{\widetilde{B}(F X)^{C}}^{C} \widetilde{B} F^{C}\right)\left(\widetilde{B} Y^{C}\right) \\
& -\hat{\omega}^{C}\left(\widetilde{B} Y^{C}\right) \widetilde{B}(F X)^{V} \\
& -\hat{\omega}^{V}\left(\widetilde{B} Y^{V}\right) \widetilde{B}(F X)^{C}+\hat{\omega}^{C}\left(\widetilde{B}(F Y)^{C}\right)\left(\widetilde{B} X^{V}\right) \\
& +\hat{\omega}^{V}\left(\widetilde{B}(F Y)^{V}\right)\left(\widetilde{B} X^{C}\right) .
\end{aligned}
$$

Interchanging $X$ and $Y$ in (4.2), we get

$$
\begin{aligned}
(4.3)\left(\hat{\nabla}_{\widetilde{B}(F Y)^{C}}^{C} \widetilde{B} F^{C}\right)\left(\widetilde{B} X^{C}\right) & =\left(\bar{\nabla}_{\widetilde{B}(F Y)^{C}}^{C} \widetilde{B} F^{C}\right)\left(\widetilde{B} X^{C}\right) \\
& -\hat{\omega}^{C}\left(\widetilde{B} X^{C}\right) \widetilde{B}(F Y)^{V}
\end{aligned}
$$




$$
\begin{aligned}
& -\hat{\omega}^{V}\left(\widetilde{B} X^{V}\right) \widetilde{B}(F Y)^{C}+\hat{\omega}^{C}\left(\widetilde{B}(F X)^{C}\right)\left(\widetilde{B} Y^{V}\right) \\
& +\hat{\omega}^{V}\left(\widetilde{B}(F X)^{V}\right)\left(\widetilde{B} Y^{C}\right) .
\end{aligned}
$$

Operating $F^{C}$ on whole equation of (4.1) and using (3.1), we get

$$
\begin{aligned}
F^{C}\left(\hat{\nabla}_{\widetilde{B} X^{C}}^{C} \widetilde{B} F^{C}\right)\left(\widetilde{B} Y^{C}\right) & =F^{C}\left(\widetilde{\nabla}_{\widetilde{B} X^{C}}^{C} \widetilde{B} F^{C}\right)\left(\widetilde{B}(F Y)^{C}\right) \\
& -\hat{\omega}^{C}\left(\widetilde{B} Y^{C}\right) \widetilde{B}(F X)^{V}-\hat{\omega}^{V}\left(\widetilde{B} Y^{V}\right) \widetilde{B}(F X)^{C} \\
& +\hat{\omega}^{C}\left(\widetilde{B}(F Y)^{C}\right)\left(\widetilde{B} X^{V}\right) \\
& +\hat{\omega}^{V}\left(\widetilde{B}(F Y)^{V}\right)\left(\widetilde{B} X^{C}\right) .
\end{aligned}
$$

Interchanging $X$ and $Y$ in (4.2), we obtain

$$
\begin{aligned}
(4.5) F^{C}\left(\hat{\nabla}_{\widetilde{B} Y^{C}}^{C} \widetilde{B} F^{C}\right)\left(\widetilde{B} X^{C}\right) & =F^{C}\left(\bar{\nabla}_{\widetilde{B} Y^{C}}^{C} \widetilde{B} F^{C}\right)\left(\widetilde{B}(F X)^{C}\right) \\
& -\hat{\omega}^{C}\left(\widetilde{B} X^{C}\right) \widetilde{B}(F Y)^{V}-\hat{\omega}^{V}\left(\widetilde{B} X^{V}\right) \widetilde{B}(F Y)^{C} \\
& +\hat{\omega}^{C}\left(\widetilde{B}(F X)^{C}\right)\left(\widetilde{B} Y^{V}\right) \\
& +\hat{\omega}^{V}\left(\widetilde{B}(F X)^{V}\right)\left(\widetilde{B} Y^{C}\right) .
\end{aligned}
$$

The Nijenhuis Tensor in an almost Hermitian manifold is given by

$$
\begin{aligned}
\bar{N}\left(X^{C}, Y^{C}\right) & =\left(\bar{\nabla}_{\widetilde{B}(F X)^{C}}^{C} \widetilde{B} F^{C}\right) \widetilde{B} Y^{C}-\left(\bar{\nabla}_{\widetilde{B}(F Y)^{C}}^{C} \widetilde{B} F^{C}\right) \widetilde{B} X^{C} \\
& -\widetilde{B} F^{C}\left(\bar{\nabla}_{\widetilde{B} X^{C}}^{C} \widetilde{B} F^{C}\right) \widetilde{B} Y^{C}+\widetilde{B} F^{C}\left(\bar{\nabla}_{\widetilde{B} Y^{C}}^{C} \widetilde{B} F^{C}\right) \widetilde{B} X^{C}
\end{aligned}
$$

In view of (4.2), (4.3), (4.4), (4.5) and (4.6)

$$
\begin{aligned}
\bar{N}\left(X^{C}, Y^{C}\right) & =\left(\hat{\nabla}_{\widetilde{B}(F X)^{C}}^{C} \widetilde{B} F^{C}\right) \widetilde{B} Y^{C}-\left(\hat{\nabla}_{\widetilde{B}(F Y)^{C}}^{C} \widetilde{B} F^{C}\right) \widetilde{B} X^{C} \\
& -\widetilde{B} F^{C}\left(\hat{\nabla}_{\widetilde{B} X^{C}}^{C} \widetilde{B} F^{C}\right) \widetilde{B} Y^{C}+\widetilde{B} F^{C}\left(\hat{\nabla}_{\widetilde{B} Y^{C}}^{C} \widetilde{B} F^{C}\right) \widetilde{B} X^{C}
\end{aligned}
$$

is Nijenhuis tensor of connection $\hat{\nabla}^{C}$.

$$
\bar{N}\left(X^{C}, Y^{C}\right)=\hat{N}\left(X^{C}, Y^{C}\right),
$$

where

$$
\begin{aligned}
\hat{N}\left(X^{C}, Y^{C}\right) & =\left(\hat{\nabla}_{\widetilde{B}(F X)^{C}}^{C} \widetilde{B} F^{C}\right) \widetilde{B} Y^{C}-\left(\hat{\nabla}_{\widetilde{B}(F Y)^{C}}^{C} \widetilde{B} F^{C}\right) \widetilde{B} X^{C} \\
& -\widetilde{B} F^{C}\left(\hat{\nabla}_{\widetilde{B} X^{C}}^{C} \widetilde{B} F^{C}\right) \widetilde{B} Y^{C}+\widetilde{B} F^{C}\left(\hat{\nabla}_{\widetilde{B} Y^{C}}^{C} \widetilde{B} F^{C}\right) \widetilde{B} X^{C} .
\end{aligned}
$$

Corollary 4.2. An almost Hermitian manifold $M$ admits a quarter-symmetric non-metric connection $\bar{\nabla}^{C}$ with respect to the Riemannian connection $\hat{\nabla}^{C}$ in $\left(T M, \hat{g}^{C}\right.$ to be a Hermitian manifold if the Nijenhuis tensor of connection $\bar{\nabla}^{C}$ vanishes, i.e. $\hat{N}\left(X^{C}, Y^{C}\right)$. 
Corollary 4.3. On a Kaehler manifold, Nijenhuis tensor with respect to the quarter-symmetric non-metric connection $\bar{\nabla}^{C}$ vanishes, i.e. $\hat{N}\left(X^{C}, Y^{C}\right)$.

Theorem 4.4. On a Kaehler manifold, Nijenhuis tensor with respect to the quarter-symmetric non-metric connection $\bar{\nabla}^{C}$ satisfies the following relations:

$(a)\left(\bar{\nabla}_{\widetilde{B}(F X)^{C}}^{C} \widetilde{B} F^{C}\right)\left(\widetilde{B}(F Y)^{C}=\left(\hat{\nabla}_{\widetilde{B} X^{C}}^{C} \widetilde{B} F^{C}\right)\left(\widetilde{B} Y^{C}\right)\right.$ i.e. $\left(\bar{\nabla}_{\widetilde{B} X^{C}}^{C} \widetilde{B} F^{C}\right)\left(\widetilde{B} Y^{C}\right)$

is hybrid in both slots.

$$
(b)\left(\bar{\nabla}_{\widetilde{B} X^{C}}^{C} \widetilde{B} F^{C}\right)\left(\widetilde{B} Y^{C}\right)=0 \Leftrightarrow \hat{\omega}^{C}\left(\widetilde{B} Y^{C}\right)=0, \hat{\omega}^{C}\left(\widetilde{B} Y^{C}\right)=0 .
$$

Proof. In view of (3.3), (3.15) becomes

$$
\begin{aligned}
\left(\bar{\nabla}_{\widetilde{B} X^{C}}^{C} \widetilde{B} F^{C}\right)\left(\widetilde{B} Y^{C}\right) & =\hat{\omega}^{C}\left(\widetilde{B} Y^{C}\right) \widetilde{B} X^{V}+\hat{\omega}^{V}\left(\widetilde{B} Y^{V}\right) \widetilde{B} X^{C} \\
& +\hat{\omega}^{C}\left(\widetilde{B}(F Y)^{C}\right)\left(\widetilde{B}(F X)^{V}\right) \\
\cdot & +\hat{\omega}^{V}\left(\widetilde{B}(F Y)^{V}\right)\left(\widetilde{B}(F X)^{C}\right)
\end{aligned}
$$

Substituting FX in place of $\mathrm{X}$ and FY in the place of $\mathrm{Y}$ in (3.15) and using (3.15), we can find

$$
\begin{aligned}
\left(\bar{\nabla}_{\widetilde{B}(F X)^{C}}^{C} \widetilde{B} F^{C}\right)\left(\widetilde{B}(F Y)^{C}\right) & =\hat{\omega}^{C}\left(\widetilde{B}(F Y)^{C}\right) \widetilde{B}(F X)^{V} \\
& +\hat{\omega}^{C}\left(\widetilde{B} Y^{C}\right)\left(\widetilde{B} X^{V}\right) \\
& +\hat{\omega}^{V}\left(\widetilde{B}(F Y)^{V}\right) \widetilde{B}(F X)^{C} \\
& +\hat{\omega}^{V}\left(\widetilde{B} Y^{V}\right)\left(\widetilde{B} X^{C}\right)
\end{aligned}
$$

in consequence of (4.9) and (4.10), we can find (4.8).

$$
\begin{aligned}
& \text { Again, if }\left(\bar{\nabla}_{\widetilde{B}(F X)^{C}}^{C} \widetilde{B} F^{C}\right)\left(\widetilde{B}(F Y)^{C}\right)=0 \text {, then (4.9) gives } \\
& \qquad \begin{array}{r}
\hat{\omega}^{C}\left(\widetilde{B}(F Y)^{C}\right) \widetilde{B}(F X)^{V}+\hat{\omega}^{V}\left(\widetilde{B}(F Y)^{V}\right) \widetilde{B}(F X)^{C}+\hat{\omega}^{C}\left(\widetilde{B} Y^{C}\right)\left(\widetilde{B} X^{V}\right) \\
+\hat{\omega}^{V}\left(\widetilde{B} Y^{V}\right)\left(\widetilde{B} X^{C}\right)=0 .
\end{array}
\end{aligned}
$$

If $X$ and $F X$ are linearly independent, hence

$$
\hat{\omega}^{C}\left(\widetilde{B} Y^{C}\right)=0, \hat{\omega}^{C}\left(\widetilde{B} Y^{C}\right)=0,
$$

which proves the first part of the statement. The converse part is obvious. 


\section{Example}

Let $V^{4}$ be a real vector space with a chosen basis $\left\{e_{1}, e_{2}, e_{3}, e_{4}\right\}$. Let $\ell$ denote the Lie algebra. Let $G$ be a real connected four-dimensional Lie group and constructed with left-invariant an almost Hermitian structure $\hat{F}^{C}$ by

$$
\hat{F}^{C} e_{1}=-e_{3}, \hat{F}^{C} e_{2}=-e_{4}, \hat{F}^{C} e_{3}=e_{1}, \hat{F}^{C} e_{4}=-e_{2},
$$

Define a left invariant non-singular Hermitian metric $g$ in $G$ by

$$
\begin{gathered}
\hat{g}^{C}\left(e_{1}, e_{1}\right)=\hat{g}^{C}\left(e_{2}, e_{2}\right)=\hat{g}^{C}\left(e_{3}, e_{3}\right)=\hat{g}^{C}\left(e_{4}, e_{4}\right)=1, \\
\hat{g}^{C}\left(e_{i}, e_{j}\right)=0, i \neq j, i, j=1,2,3,4 .
\end{gathered}
$$

Thus, in consequence of (3.1), (3.2), (5.1), (5.2),

$\left\{G, \phi, \xi_{p}, \eta^{p}, g\right\}$ is an almost Hermitian manifold on a tangent bundle.

\section{RE F E R E N C E S}

1. N. Agashe and M. R. Chafle A semi-symmetric non-metric connection on a Riemannian manifold. Indian J. pure Applied Math. 23 (6), (1992), 399-409.

2. B. B. Chaturvedi and P. N. PANDEY A semi-symmetric non-metric connection on Kaehler manifold. Differential Geometry-Dynamical Systems. 10 (2008), 86-90.

3. L. S. DAS and M. N. I. KhAN Almost r-contact structures on the Tangent bundle. Differential Geometry-Dynamical Systems. 7 (2005), 34-41.

4. S. GolaB On semi-symmetric and quarter symmetric linear connection. Tensor N.S. 29 (1975), 249-254.

5. H. A. HaYden Subspace of a space with torsion. Proc. London Math Soc. 34 (1932), 27-50.

6. S. IANUS and C. UdRite On the tangent bundle of a differentiable manifold, Stud. Cerc. Math. 22 (1970), 599-611.

7. M. N. I. KHAN A quarter-symmetric semi-metric connection on the Sasakian manifold, Tensor N.S. 68 (2007), 154-157.

8. M. N. I. KHAN Lifts of hypersurfaces with Quarter -symmetric semi-metric connection to tangent bundles. Afr. Mat. 25(2) (2014), 475-482.

9. M. N. I. Khan Lift of semi-symmetric non-metric connection on a Kahler manifold. Afr. Mat. 27 (3) (2016), 345-352.

10. M. N. I. KhAN and J. B. JUN Lorentzian almost r-para-contact structure in tangent bundle. Journal of the Chungcheong Mathematical Society. 27(1) (2014), 29-34.

11. M. TANi Prolongations of hypersurfaces of tangent bundles. Kodai Math. Semp. Rep. 21 (1969), 85-96. 
12. M. Tekkoyun and S. CiveleK On lifts of structures on complex manifolds. Differential Geometry-Dynamical Systems. 5(1) (2008), 373-382.

13. K. YANO and S. IshiHARA Tangent and Cotangent Bundles. Marcel Dekker Inc. New York. 1973.

14. K. Yano and S. Kobayashi Prolongations of tensorfields and connections to tangent bundle. J. Math. Soc. Japan. 18 (1966), 194-210.

Mohammad Nazrul Islam Khan

Department of Computer Engineering

College of Computer

Qassim University

P. O. Box 6688

Buraidah-51452, Saudia Arabia

m.nazrul@edu.qu.sa, mnazrul@rediffmail.com 